\title{
A Complicated Hospitalization Following Dilute Ammonium Chloride Ingestion
}

\author{
Kendra Hammond, $M D^{a}$, Tiffany Graybill, DO a , Susannah E. Spiess, MD ${ }^{b, c}$, Jenny Lu, MD ${ }^{d}$ \\ Jerrold B. Leikin, $M D^{e}$
}

aDepartment of Medicine, Evanston Hospital, Evanston, IL

bDivision of Gastroenterology, NorthShore University HealthSystem, Glenview, IL

cNorthwestern University Medical School, Evanston, IL

dToxikon Consortium, John Stroger, Jr. Hospital, Chicago, IL

eNorthShore University HealthSystem OMEGA, Glenbrook Hospital, Glenview, IL

\section{ABSTRACT}

Introduction: Unintentional ingestions of dilute $(<7.5 \%)$ cleaning solutions containing ammonium chloride typically do not cause serious harm. We present a case of an intentional ingestion of a dilute ammonium chloride solution resulting in significant morbidity.

Case Report: A 60-year-old woman with bipolar disorder presented one hour after an intentional ingestion of approximately 15 fluid ounces $(500 \mathrm{~mL})$ of an algae and odor humidifier treatment containing a total of $2.25 \%$ ethyl ammonium chloride. Initial complaints included nausea with a single episode of nonbilious, nonbloody emesis, mild shortness of breath, and chest and epigastric pain. Physical exam was remarkable for bilateral wheezing and epigastric tenderness. An emergent endoscopy demonstrated a Grade $2 \mathrm{~b}$ caustic injury in the esophagus and a Grade $3 \mathrm{~b}$ injury in the stomach. Due to persistent cough, copious oral secretions, and worsening hoarseness, the patient was intubated and admitted to the ICU. Her course was complicated by mild hypotension, nonanion gap metabolic acidosis, and oliguria treated successfully with intravenous (IV) fluids. She also developed bilateral pneumonias later in the hospital course. Bedside bronchoscopy showed laryngeal edema and mucosal injury to the segmental level. The patient underwent tracheostomy on hospital day 6. An upper GI swallow study revealed poor esophageal motility in the mid- to lower third of the esophagus. The patient gradually tolerated oral fluids and on hospital day 20 had her tracheostomy tube removed. The patient was subsequently transferred to the psychiatric ward on hospital day 22 .

Conclusion: Intentional ingestions of dilute ammonium chloride solutions can cause serious injury to the gastrointestinal tract and pulmonary systems, which can result in a complicated and prolonged hospitalization.

\section{INTRODUCTION}

Caustic exposures to household and industrial products such as cleaners, germicides, and detergents are regularly encountered by poison centers and emergency department physicians. In the United States, the pediatric population composes a large number of the unintentional exposures. The incidence of pediatric exposures, however, dramatically decreased as a result of child-resistant pack-

Keywords: ammonium chloride, caustic, acid

Notes: Accepted for presentation to the North American Congress of Clinical Toxicology, Toronto, Canada, September 2008.

There was no outside funding of any kind used for this study.

The authors have no potential financial conflicts of interest to report.

Corresponding Author: Jerrold B. Leikin MD, NorthShore University HealthSystem-OMEGA, 2150 Pfingsten Road, Suite 3000, Glenview IL 60026. Email: jleikin@northshore.org 
aging legislation and poison prevention education. Although they comprise only a minority of caustic ingestions, intentional ingestions by adults tend to be more serious and more likely to require treatment [1]. Acids and alkalis both cause functional and histological tissue damage on contact, alkalis through liquefaction necrosis and acids through coagulative-type tissue necrosis $[1,2]$. Factors affecting the degree of injury may include the duration of contact, volume and concentration, $\mathrm{pH}$ of the substance, physical state of the corrosive (solid versus liquid) and the titratable acid or alkaline reserve (TAR)[1].

Ammonium chloride is found in many hospital cleaning products and germicides. Concentrated (10-15\%) solutions are known to cause severe burns of the upper gastrointestinal tract after only a few milliliters. Lower concentrations $(<7.5 \%)$ usually do not lead to significant mucosal injury [3]. These relatively more dilute cleaners are generally considered to be less caustic. We describe a case in which a moderate-volume ingestion of a relatively dilute, weak acid resulting in significant caustic injury to the upper pulmonary tree and gastrointestinal tract required intensive and multidisciplinary medical intervention.

\section{CASE REPORT}

A 60-year-old woman with a history of bipolar disorder and previous suicide attempts presented to the emergency department (ED) via ambulance approximately 1 hour following the intentional ingestion of a caustic substance in a suicide attempt. She stated that she had ingested approximately 15 fluid ounces (500 $\mathrm{mL}$ ) of Best Air Bacteriostatic Algae and Odor Humidifier Treatment, containing a total of $2.25 \%$ ammonium chloride $(1.125 \%$ n-alkyl dimethyl benzyl ammonium chloride $+1.125 \%$ n-alkyl dimethyl ethylbenzyl ammonium chloride). Her initial complaints included nausea, nonbilious, nonbloody emesis, mild shortness of breath, chest pain, and epigastric abdominal pain. Her frequent coughing produced copious amounts of white, frothy sputum and she had multiple loose brown stools while in the ED. Her initial vital signs were: temperature, 36.7C; blood pressure, 152/78; pulse, $114 / \mathrm{min}$; and respirations $22 / \mathrm{min}$. Her oxygen saturation was $91 \%$ on room air, which improved to $97 \%$ with the placement of 6 L of O2 by nasal cannula. Physical examination revealed an alert, but disheveled-appearing woman with intermittent coughing. Bilateral wheezing was found on lung auscultation. Cardiac exam demonstrated normal heart tones, but mild tachycardia. Abdominal exam was notable for mild epigastric tenderness without guarding or rebound. The neurological exam was grossly intact without focal deficit. Examination of the oropharynx revealed only dryness of the mucous membranes. No discrete ulcerations or erythema were noted. Laboratory analysis demonstrated a leukocytosis with WBC of 3.6 thou $/ \mathrm{mm}^{3}$ with the remainder of the CBC within normal limits. Her metabolic panel demonstrated mild hypokalemia of $3.4 \mathrm{mEq} / \mathrm{L}$, with normal renal function and an anion gap of 11 in the setting of a normal albumin. Lactic acid was $2.1 \mathrm{mmol} / \mathrm{L}$.
Upon consultation by the ED physician, the poison center recommended supportive care including airway management as needed and, based on her symptomatic presentation, emergent evaluation of the esophagus and upper gastrointestinal tract to determine the extent of caustic injury. Oxygen and intravenous (IV) fluids were provided and the patient was maintained in a fasting state until further evaluation. Within 1 hour of presentation to the ED, an emergent esophagogastroduodenoscopy (EGD) was performed, demonstrating a Grade $2 \mathrm{~b}$ caustic injury in the esophagus and Grade 3 injury in the stomach (Figures 1 and 2).

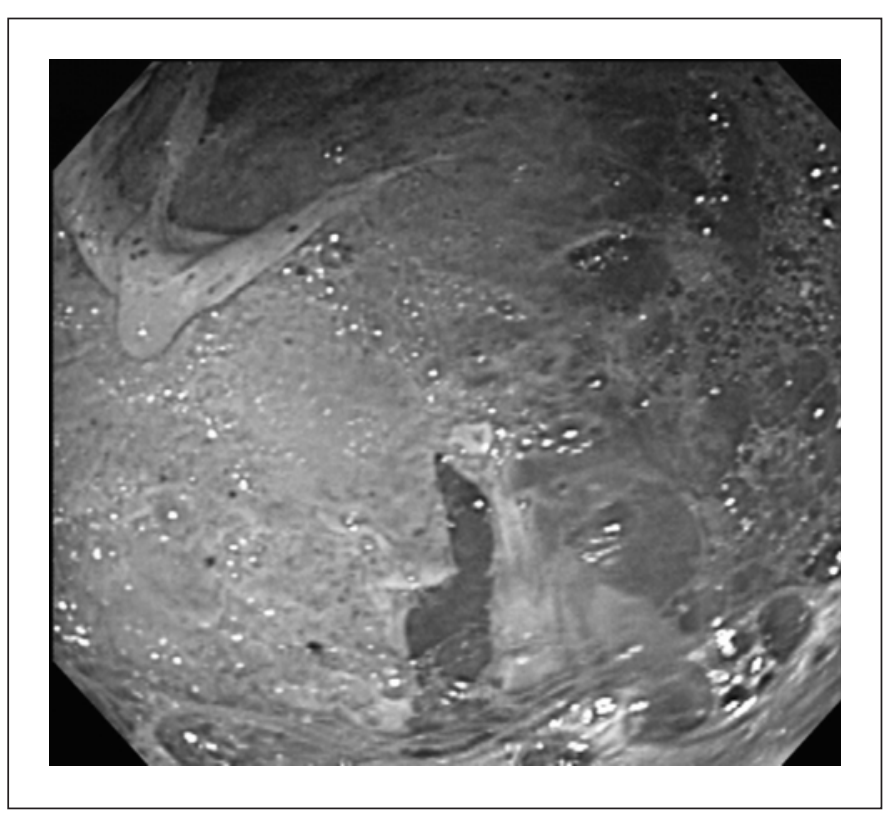

Figure 1: Grade $2 b$ caustic injury of esophagus, with superficial exudates and denuded mucosa.

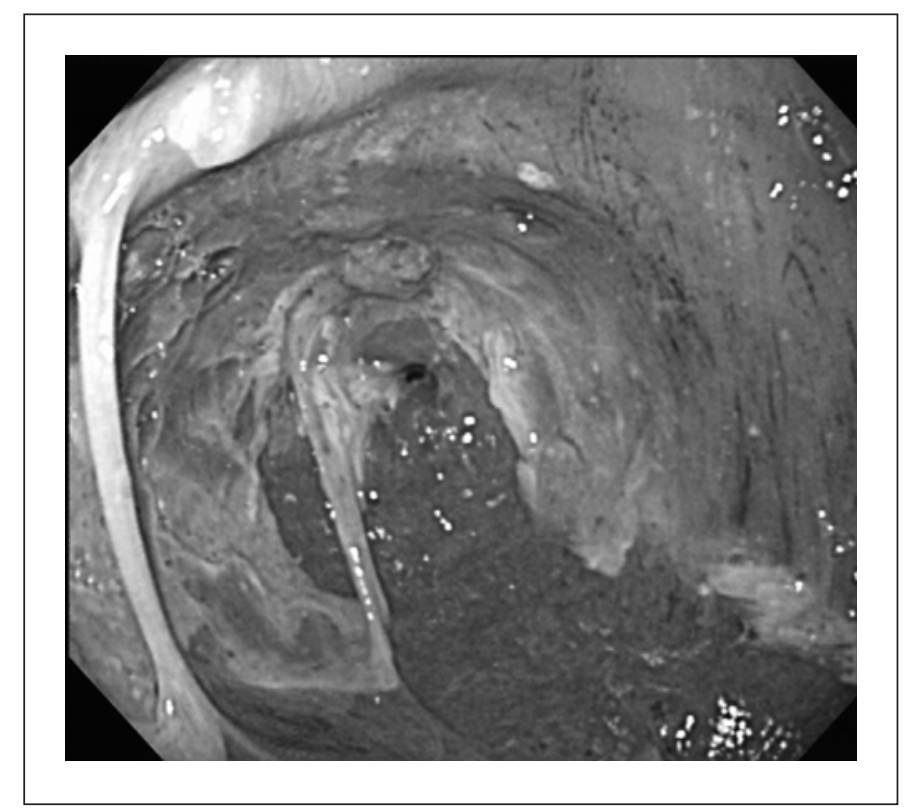

Figure 2: Grade 3 caustic injury of stomach, with dusky appearance and sloughing of mucosa. 
No significant damage was seen in the duodenal bulb. The patient was given midazolam $1.5 \mathrm{mg}$ (IV) and meperidine $25 \mathrm{mg}$ (IV) for procedural sedation for the endoscopy, which was performed without complications. Following the EGD, the patient continued to have copious oral secretions and worsening hoarseness, increasing the concern regarding caustic tracheal injury and worsening upper airway edema. She was consequently intubated for airway protection and transferred to the Medical Intensive Care Unit (MICU) for close monitoring and supportive care. Empiric antibiotics were initiated for possible aspiration pneumonia and a proton pump inhibitor drip was administered to prevent stress-related mucosal disease. The cardiothoracic surgical service was consulted in the ED following EGD in case a perforation injury developed.

In the ICU, the patient's condition consisted of a worsening diarrhea associated with a mild, associated hypotension (systolic blood pressure in the 90s $\mathrm{mmHg}$ ) and oliguria. She received several liters of IV fluids to maintain a steady urine output and stable blood pressure. No significant change in renal function was noted by laboratory analysis during this time. The patient remained intubated and sedated. By hospital day 2, she developed a mild primary metabolic acidosis ( $\mathrm{pH} 7.34$ ), with normal anion gap and with a superimposed respiratory acidosis that resolved over the course of the next several days. No elevation of lactate was noted. On the third hospital day, a bedside bronchoscopy revealed laryngeal edema with mucosal injury down to the segmental level, for which IV steroid therapy was initiated. Due to a persistent endotracheal cuff leak, she underwent a tracheostomy and was started on parenteral nutrition on hospital day 6. Enteral nutrition was withheld secondary to the degree of injury visualized on endoscopy and concern for perforation.

The patient's ICU course was further complicated by the development of bilateral pneumonias, for which antibiotic coverage was broadened. The following day she was gradually weaned off sedation and mechanical ventilation and was transferred to the general medical floor. With supportive care, the patient's bilateral pneumonias and general condition improved. She remained on parenteral nutrition during this time as there remained a concern for possible esophageal stricture and/or pyloric stenosis given the degree of injury seen on her admission EGD. The placement of a G-tube was not considered an option based on the extent of her gastric injury. A barium swallow was subsequently performed, demonstrating poor esophageal motility in the mid- to lower third of the esophagus, with no evidence of stricture or ulcerations. She was then started on a clear-liquid diet and gradually advanced to a low-fat, low-fiber diet, which she tolerated well. During this time she was closely observed for any signs of perforation. Her tracheostomy was removed on hospital day 20 without complication and she was eventually transferred to an inpatient psychiatric ward on hospital day 22.

\section{DISCUSSION}

Exposures to both acids and alkalis through ingestions can result in a wide variety of presentations, severity, and complications [4].
Acid ingestions can spare the esophagus but can result in corrosive-induced gastric outlet obstruction [5-7]. Other than dilute hydrofluoric acid ( $<8 \%$ concentration), systemic and metabolic abnormalities rarely occur.

Quaternary ammonium compounds were first used in 1935. The formulation in the product ingested by our patient contains 2 dual quaternary ammonium compounds (containing a total of 2.25\% ammonium chloride [1.125\% n-alkyl dimethyl benzyl ammonium chloride $+1.125 \%$ n-alkyl dimethyl ethylbenzyl ammonium chloride]) that are potent bacteriostatic solutions commonly found in disinfectants and sanitizers. Ammonium chloride is a cationic detergent that is usually not considered to have severe irritant properties. The $\mathrm{pH}$ of ammonium chloride ranges from 5 to 5.5 at $25 \mathrm{C}$ (1-10\% solution), which makes this compound a relatively weak acid solution [9]. Solutions of 5-10\% of ammonium chloride have not been demonstrated to cause ocular irritation upon contact [10]. Fatal ingestions due to $0.2 \%$ concentration may have pHs high enough to significantly increase the risk of serious esophageal burns, although other factors also contribute to risk of esophageal damage [11]. Fatalities from ingestions of aqueous solutions of benzalkonium chloride have rarely been reported and usually involve products of greater than $10 \%$ concentration [12]. Complications from these types of ingestions include-although rare-caustic burns to the mouth and esophagus, transaminase elevations, metabolic acidosis, and central nervous system depression. Muscle paralysis (which was not seen in our patient) following ingestions of quaternary ammonium compounds has been described and is presumed to be likely due to cholinesterase inhibition at the neuromuscular junction [12].

Immediate management concerns of caustic injuries include patient decontamination, assessing and securing the airway as necessary, evaluating the extent of injuries from the physical exam, vital signs, obtaining information regarding the caustic ingestion, and early involvement of surgical and medical specialties for endoscopic evaluation and possible surgical intervention. Volume losses should be replaced judiciously. If the patient is asymptomatic, and the amount of accidental ingestion determined to be small in volume, endoscopic evaluation of the upper gastrointestinal tract may not be necessary. Radiological and laboratory studies that should be considered include a chest radiograph, arterial blood gas, lactate, blood type and cross-match, complete blood count, chemistry, coagulation parameters, and urinalysis. Any suggestion of perforation mandates immediate surgical intervention.

Several studies have attempted to define predictors of injury, as patients with grade 2 and 3 esophageal injuries have higher risks of developing severe complications. One study suggested that drooling, vomiting, and stridor could be used to predict esophageal injury, while another found that drooling, buccal mucosal burns, and white blood cell count were significant independent predictors of the degree of gastrointestinal injury (13-15). Other studies, however, could not identify signs or symptoms that could be used to reliably predict degree of injury $[16,17]$. The presence or absence of oropharyngeal burns identified on physical exami- 
nation is not a reliable predictor of distal esophagogastric injury [15-18].

The timing of the EGD is controversial, with some experts recommending performing it emergently while others suggesting waiting 48-72 hours to assess the full extent of the injury $[13,14,19]$. Endoscopic grading of caustic injuries is based on 4 grades: Grade 1, edema and erythema; Grade 2 ( $a$ indicates linear, $b$ indicates circumferential) hemorrhages, erosions, blisters, superficial ulcers, and exudates; Grade 3, multiple deep brownish-black or gray ulcers; Grade 4, perforation $[14,19,20]$.

Caustic tracheobronchitis can also be seen, as was present in our patient. Causes of tracheobronchitis include aspiration of the caustic substance during the initial ingestion or during subsequent emesis, injury to the tracheobronchial tree through necrotic extension from a significantly-injured esophagus, or through involvement of the mediastinum [21]. Similar to the injuries seen in the upper gastrointestinal tract, caustic tracheobronchitis can lead to fibrosis and clinically significant stenosis ][21-24].

The treatment goals are to prevent perforation and avoid stricture formation of the esophagus and stomach. Multiple therapeutic modalities have been described to achieve this; however, many have been unproven. For example, since alkali injuries usually occur rapidly, attempts to neutralize them would be unsuccessful and may even lead to aspiration [13,19]. Use of corticosteroids is controversial. Animal studies show a decrease in incidence of esophageal strictures; however, human prospective, randomized, controlled trials in children show that corticosteroids do not prevent strictures $[23,24]$. As far as the use of antibiotics, no prospective human studies are available.

The late complications of caustic ingestions in the gastrointestinal tract include esophageal stricture formation, antral stenosis, squamous cell carcinoma of the esophagus, and carcinoma of the stomach. The rate of stricture formation appears to depend on degree of injury, with $30 \%$ of persons with third-degree burns and $15-30 \%$ of those with second-degree burns developing strictures [26]. Other less common complications include tracheal stenosis, tracheobronchial fistula, and aortoenteric or gastrocolic fistulization $[27,28]$. The mortality rate after caustic ingestions is $1-6 \%$ and is usually due to mediastinitis, peritonitis, or malnutrition $[6,29]$.

\section{CONCLUSION}

Although unintentional exposures of dilute ammonium compounds generally do not cause serious injury, severe gastrointestinal and respiratory complications can occur in rare instances, depending on the circumstances. The intentional nature of a suicidal ingestion can undoubtedly contribute to the severity of injury, as in this case. Management and treatment strategies should focus on immediate stabilization of airway and hemodynamics, followed by emergent endoscopy, if indicated, to assess the extent of injury. Gastrointestinal and surgical specialties should be involved in a timely fashion if the clinical picture suggests a potentially seriously-injured patient. General treatment principles for caustic exposures should be applied to all cases of ingestion, until the patient can be reliably cleared. Although generally less frequently encountered than with more concentrated products, dilute compounds still exhibit the potential to cause serious damage to the gastrointestinal and respiratory systems. We present here a case of an intentional ingestion of dilute ammonium chloride compound that resulted in a complicated hospital course.

\section{REFERENCES}

1. Ramasamy K, Gumaste VV. Corrosive ingestion in adults. J Clin Gastroenterol. 2003;37(2):119-124.

2. Howell, JM. Alkaline ingestions. Ann Emerg Med. 1986;15(7):820-825.

3. Tiess D, Nagel KH. Contribution to the morphology and analysis of invert soap poisoning: 2 cases of fatal acute poisoning caused by oral consumption of the disinfectant C4. Arch Toxicol. 1967;22(5):333-348.

4. Dilawari JB, Singh S, Rao PN, et al. Corrosive acid ingestion in man-a clinical and endoscopic study. Gut. 1984;25(2): 183-187.

5. Ali N, Eni UE. Corrosive-induced gastric outlet obstruction without esophageal involvement: a case report. Niger J Med. 2005;14(4):436-438.

6. Kaushik R, Singh R, Sharma R, et al. Corrosive-induced gastric outlet obstruction. Yonsei Med J. 2003;44(6):991-994.

7. Ozcan C, Ergun O, Sen T, et al. Gastric outlet obstruction secondary to acid ingestion in children. J Pedriatr Surg. 2004;39(11):1651-1653.

8. Kao WF, Dart RC, Kuffner E, et al. Ingestion of low concentration hydrofluoric acid: an insidious and potentially fatal poisoning. Ann Emerg Med. 1999;31(1):35-41.

9. Budavari S. The Merck Index. 11th ed. Rahway, NJ: Merck \& Co.; 1989:83.

10. Grant WW, Schuman JS. Toxicology of the Eye. 4th ed. Springfield, IL: Charles C. Thomas; 1993.

11. Howell JM. Alkalinity of non-industrial cleaning products and the likelihood of producing significant esophageal burns. Am J of Emerg Med. 1991;9(6):560-562.

12. Hitosugi M, Maruyama, K, Takatsu A. A case of fatal benzalkonium chloride poisoning. Int J Legal Med. 1998;111(5): 265-266.

13. Rao R, Hoffman RS. Caustics and Batteries. In Goldfrank LR, eds. Goldfrank's Toxicologic Emergencies. 7th ed. McGraw-Hill, Professional; 2002:1323-1340.

14. Havanond C, Havanond P. Initial signs and symptoms as prognostic indicators of severe gastrointestinal tract injury due to corrosive ingestion. J Emerg Med. 2007;33(4):349-353.

15. Crain EF, Gershel JC, Mezey AP. Caustic ingestions: symptoms as predictors of esophageal ingestions. Emerg Med Clin North Am. 1984;2(1):77-86.

16. Gorman RF, Khin-Mang-Oyi MT, Klein-Schwartz W, et al. Initial symptoms as predictors of esophageal injury in alkaline corrosive ingestions. Am J Emerg Med. 1992;10(3):189-194. 
17. Gaudreau HP, Parent M, McGuigan MA, et al. Predictability of esophageal injury from signs and systems: a study of caustic ingestion in 378 children. Pediatrics. 1983;71(5):767-770.

18. Previtera C, Guisti F, Guglielmi M. Predictive value of visible lesions (cheeks, lips, oropharynx) in suspected caustic ingestion: may endoscopy reasonably be omitted in completely negative pediatric patients? Pediatr Emerg Care. 190;6:176-178.

19. Kikendall JW. Caustic ingestion injuries. Gastroenterol Clin North Am. 1991;20:847.

20. Zargar SA, Kochhar R, Nagi B, et al. Ingestion of strong corrosive alkalis: spectrum of injury to upper gastrointestinal tract and natural history. Am J Gastroenterol. 1992;87:337.

21. Represas C, Fernandez-Villar A, Leiro V, et AL. Caustic tracheobronchitis. J Bronchol. 2007;13(3):156-158.

22. Ray JF. Liquid caustic ingestion: a flag of caution. Arch Intern Med. 1980;140:471.

23. Rosenberg N, Kunderman PJ, Vroman L, et al. Prevention of experimental lye strictures of the esophagus by cortisone. Arch Surg. 1951;63:147.
24. Anderson KD, Rouse MR, Randolph GA. A controlled trial of corticosteroids in children with corrosive injury of the esophagus. N Engl J Med. 1990;323:637.

25. Pelclova D, Navratil T: Do corticosteroids prevent oesophageal stricture after corrosive ingestion? Toxicol Rev. 2005;24(2):125-129.

26. Gumaste VV, Dave PB. Ingestion of corrosive substances by adults. Am J Gastroenterol. 1992;87:1-5.

27. Keh SM, Onyekwelu N, McManus K, et al. Corrosive injury to upper gastrointestinal tract: still a major surgical dilemma. World J Gastroenterol. 2006;12(32):5223-5228.

28. Tohda G, Sugawa C, Gayer C, et al. [webpage on the Internet]. Clinical evaluation and management of caustic injury in the upper gastrointestinal tract in 95 adult patients in an urban medical center. [cited XXX] Surgical Endoscopy [online]. October 2007. Available from:

29. Conti S, Tesfay M, Picone P, et al. Corrosive espophageal injuries in children: A shortlived experience in Sierra Leone. Int J Pediatric Otorhinolaryngol. 2007;70(10):1597-1604. 